\title{
Estudo comparativo de práticas de promoção da saúde na atenção primária em Florianópolis, Santa Catarina, Brasil e Toronto, Ontário, Canadá
}

\section{Resumo}

Compreender as experiências de organização dos sistemas públicos de saúde universais em relação à promoção da saúde em unidades de atenção primária de Florianópolis, Santa Catarina, Brasil, e Toronto, Ontário, Canadá. Pesquisa exploratória descritiva de abordagem qualitativa realizada em unidades da atenção primária. Para a coleta de dados foram utilizadas entrevistas semiestruturadas com questões sobre as práticas de promoção, com 25 profissionais em Florianópolis, e 10, em Toronto. Os dados foram discutidos por meio de análise temática, identificando as práticas, dificuldades e facilidades da promoção da saúde. Nessas cidades, $60 \%$ dos profissionais e gestores não receberam conhecimento específico de promoção na sua formação. No que tange às habilidades promotoras de saúde, em Toronto identificou-se que os sujeitos reconhecem a autonomia e os determinantes sociais, já em Florianópolis as relacionam com a educação em saúde e participação popular. Em ambas as cidades, as práticas de promoção são direcionadas para atividades individuais e coletivas. A motivação para atuar provém da interdisciplinaridade e das demandas oriundas da população. Destaca-se a relevância da promoção, como forma de cuidado e estímulo à autonomia do indivíduo e da comunidade, considerando os determinantes sociais. Essas práticas alcançam a saúde integral da comunidade, porém, observam-se limites das equipes que ainda realizam atividades voltadas para a doença. Os recursos são escassos, necessitando de ações intersetoriais para a melhoria da qualidade de vida. A atenção à saúde está voltada para o modelo hegemônico, carecendo avançar para a concepção positiva da saúde e determinantes sociais.

Promoção da Saúde; Determinantes Sociais da Saúde; Atenção Primária à Saúde
Ivonete Teresinha Schulter Buss Heidemann 1

Camilla da Costa Cypriano 1

Denise Gastaldo 2

Suzanne Jackson 2

Carolina Gabriele Rocha 1

Eloi Fagundes 2

doi: 10.1590/0102-311X00214516

\author{
Correspondência \\ I. T. S. B. Heidemann \\ Universidade Federal de Santa Catarina. \\ Av. Servidão Natureza 160, Florianopolis, SC 88063-545, \\ Brasil. \\ ivonete.heidemann@ufsc.br \\ 1 Universidade Federal de Santa Catarina, Florianópolis, Brasil. \\ 2 University of Toronto, Toronto, Canada.
}




\section{Introdução}

A promoção da saúde é identificada como a terceira revolução da saúde pública em nivel mundial, tendo a primeira combatido as doenças infecciosas e a segunda, as doenças crônicas. A promoção da saúde, cujo foco está centrado na luta contra diversos tipos de doenças 1 , foi conceituada na Carta de Ottawa como "o processo que busca permitir que as pessoas aumentem o controle e a melhoria da saúde" 2 (p. 1). Embora a Carta permaneça proeminente nas discussões sobre a promoção da saúde em todo o mundo, esse termo provoca ambiguidades e controvérsias 3.

Nessa dicotomia, prevalecem dois discursos distintos: um com ênfase na tecnologia médica e mudança de comportamentos individuais como soluções para os problemas enfrentados 3; e outro, que busca avançar numa perspectiva emancipatória, pontecializar o principio da participação e produzir conhecimentos e informações a fim de valorizar a autonomia dos sujeitos e transformar a realidade 4 .

Apesar da compreensão divergente, vários países têm incorporado os princípios da promoção da saúde. No cenário internacional foram criadas redes colaborativas entre gestores e diferentes profissionais, estimulado o diálogo entre o governo e a população por meio da participação popular e da avaliação das ações promotoras. No Brasil, as ações de promoção da saúde emergiram como desdobramento do esforço de qualificação do Sistema Único de Saúde (SUS) e do debate sobre as relações entre desigualdades sociais. A criação da Política Nacional de Promoção da Saúde (PNPS), em 2006, e reformulada em 2014 5, ampliou os programas de promoção da saúde como Vida no Trânsito, a Rede Nacional de Prevenção das Violências, a Academia da Saúde, o Programa Saúde na Escola e também novos marcos legais para o monitoramento da propaganda de alimentos, redução do uso de álcool e do tabaco ${ }^{3}$. O processo de implantação da PNPS no Brasil teve uma trajetória longa e conflituosa, com foco preventivista, mas representou avanços na elaboração de políticas de saúde 6 .

A Carta da Ottawa enumerou inicialmente cinco estratégias essenciais para a promoção da saúde: construção de políticas públicas saudáveis; desenvolvimento de habilidades pessoais; criação de ambientes favoráveis à saúde; fortalecimento da ação comunitária; e reorientação dos serviços de saúde 2. Para o seu alcance, requerem-se ações interdisciplinares e intersetoriais que superem as abordagens preventivistas sobre as situações de saúde ${ }^{3}$. Ao se propor práticas de promoção da saúde deveriam ser incorporadas essas estratégias no processo de trabalho dos profissionais de saúde, oportunizando novas formas de produção do cuidado 7 . Embora nem todos os princípios sejam aplicáveis ao contexto da saúde, eles direcionam onde e como as inovações lideradas pelos profissionais podem ser realizadas dentro de um modelo abrangente de continuidade do cuidado e empoderamento ${ }^{8}$.

Existem várias definições que complementam as práticas de promoção da saúde, com destaque para o conceito dos Determinantes Sociais da Saúde, que, quando em sinergia, podem contribuir para uma melhor compreensão e endereçamento das iniquidades de saúde e alcance da equidade 9,10. Enquanto autores, acredita-se que a promoção da saúde deve se concentrar no engajamento comunitário e nas políticas públicas, a fim de modificar os determinantes sociais da saúde. Por meio do engajamento comunitário pode ocorrer o autocontrole das comunidades sobre as decisões que afetam diariamente as suas vidas e promovem o empoderamento, a coesão social, a solidariedade e possibilitam a diminuição das desigualdades sociais ${ }^{11}$. A promoção da saúde é relevante para o século XXI, pois os desafios, os resultados e oportunidades são evidentes, especialmente pela transição demográfica e epidemiológica, como o aumento das doenças crônicas degenerativas 12. Mas, para que ocorram mudanças os determinantes sociais da saúde devem ser abordados, visto que a promoção da saúde é limitada, sem ação efetiva nas condições de vida da população.

Considerando que este é um estudo comparativo entre duas realidades distintas, é necessário introduzir uma breve distinção entre ambas. Cita-se como pontos de destaque a Carta de Ottawa 2 , no Canadá, e a PNPS 5 no Brasil. Em ambos os países coincidem lutas políticas para a criação da atenção primária à saúde e a implementação da promoção da saúde na atenção primária.

No Brasil, o SUS se baseia no princípio da saúde como direito do cidadão e dever do Estado. Esse sistema vem se fortalecendo como um dos principais instrumentos governamentais de proteção à vida e à população, desempenhando pelo menos dois tipos de função: de saúde pública e de assistência ao processo de saúde e doença 13,14. É apresentado subdividido em diferentes níveis de atenção: hospitalar, ambulatorial e primário. A atenção primária à saúde tem a saúde da família como estratégia prioritária para a sua organização de acordo com os preceitos do SUS 15, estrutura-se na lógica de 
novas práticas setoriais, associando os trabalhos clínicos e a promoção da saúde. A Política Nacional de Atenção Primária brasileira foi introduzida pelo Ministério da Saúde em 200616.

Em Florianópolis, Santa Catarina, o sistema organizacional da atenção primária à saúde é composto por 49 unidades de saúde, subdivididas em quatro distritos sanitários, que se responsabilizam pela administração da rede em nível regional, e 12 Núcleos de Apoio à Saúde da Família (NASF), os quais oferecem cobertura a toda população, tanto central, quanto periférica, indiferentemente da classe social. Esse sistema está em processo de configuração como porta de entrada ao sistema de saúde, sendo reconhecidos os esforços para integrar a Estratégia Saúde da Família (ESF), a rede de serviços, tanto por gestores como por profissionais e famílias 17.

O sistema de saúde no Canadá é caracterizado por ser universal e financiado publicamente por meio da arrecadação de impostos e taxas, e destes, aproximadamente $70 \%$ são destinados para o setor saúde. A regulação da assistência médica fica sob a responsabilidade de cada província e território, que têm autonomia para decidir os serviços a ofertar (por exemplo, há mais cuidados domiciliares em algumas províncias), mas todo cidadão deve ter assistência médica 18.

A cobertura universal de assistência médica foi introduzida no Canadá, na província de Saskatchewan, em 1962. Durante aquele acontecimento, a medicina organizada foi altamente oposta à nova política de saúde, considerando-a uma ameaça potencial à tomada de decisão clínica e à autonomia profissional sobre o faturamento das consultas dos pacientes, o que culminou em uma greve dos médicos por 23 dias. Essa terminou somente com o acordo de Saskatoon, que protegeu o status desses profissionais como contratantes independentes, pagos por uma taxa de serviço dentro do novo sistema. Pelo acordo, eles negociariam os aumentos das taxas diretamente com o governo provincial e teriam uma influência considerável na decisão de quais novos serviços ou procedimentos de saúde deveriam ser incluídos na gama de serviços de assistência médica universalmente cobertos 19 .

Durante parte do século XX, os cuidados primários foram em grande parte prestados por médicos que trabalhavam num consultório individual, ou em pequenos grupos, partilhando instalações e despesas gerais, mas todas as consultas eram pagas pelo governo, não havendo clínica privada. Apesar da melhoria do acesso, o foco era nos serviços médicos básicos com poucos incentivos para a prevenção de doenças e promoção da saúde, o que desestimulou a adoção dos princípios da atenção primária à saúde e o envolvimento de outras profissões na prestação de cuidados primários 19.

No início dos anos 2000, uma nova política de saúde surgiu no Canadá em resposta a uma crescente insatisfação profissional e da opinião pública com os cuidados primários oferecidos, que culminou em cinco objetivos nacionais de reforma: aumentar o acesso aos cuidados primários; aumentar a ênfase na promoção da saúde e na prevenção de doenças crônicas; expandir a gestão dos cuidados essenciais; instituir equipes de cuidados multidisciplinares; e facilitar a integração com outros serviços de saúde 20.

O governo de Ontário (província em que está situada a cidade de Toronto), a partir de 2002, lançou uma série de modelos de cuidados primários para aumentar o acesso, melhorar a qualidade de vida e a prestação de serviços à população ${ }^{19}$. Essa reforma se baseou fortemente em mudanças na remuneração dos médicos e no fornecimento de incentivos e bônus de desempenho para as equipes que atingissem seus objetivos, expansão das equipes multiprofissionais de atendimentos primários, incluindo enfermeiros, assistentes sociais, nutricionistas, farmacêuticos, promotores de saúde 20 . A atenção primária à saúde em Toronto, atualmente, é composta por uma rede de 37 community health centers (CHC). Cada centro de saúde é responsável por uma região específica e tem uma estrutura e característica organizacional próprias, com uma variação considerável em quantidade e distribuição de profissionais e financiamento 21 .

Nesse contexto, foram inqueridos os profissionais dos $\mathrm{CHC}$ de Toronto e das unidades de saúde de Florianópolis sobre: "Qual é a compreensão das práticas de promoção da saúde?”; “Como desenvolvem, executam e avaliam o seu trabalho?"; e "Quais são as principais facilidades e dificuldades?”.

Para responder a tais questionamentos, este estudo buscou comparar as práticas de promoção da saúde desenvolvidas nesses centros de saúde, com o objetivo de compreender as formas de promoção da saúde nos dois países, cujos sistemas universais de saúde apresentam diferentes níveis socioeconômicos. 


\section{Método}

Esta pesquisa qualitativa de cunho comparativo foi realizada em Toronto, Ontário, Canadá, em 2014, por conta de um estudo de pós-doutorado de uma das autoras (I.T.S.B.H.), com a participação de coautores (D.G., S.J. e E.F.), e de Jennifer Ko. A seguir, na cidade de Florianópolis, Santa Catarina, Brasil, replicou-se o mesmo estudo em 2015, por meio de uma pesquisa de mestrado (C.C.C.) com a participação de coautores (I.T.S.B.H. e C.G.R.). Os dados obtidos posteriormente foram comparados, tendo em vista que os dois países enunciaram políticas públicas que contemplam a promoção da saúde. Influenciaram essa decisão: a PNPS no Brasil; a origem da promoção da saúde, na década de 1970, em países desenvolvidos como o Canadá; o reconhecimento da sua relevância internacional; e o pressuposto de que este estudo poderia fornecer subsidios ainda não abordados no contexto do conhecimento atual. Além disso, as cidades selecionadas implantaram a atenção primária à saúde, sendo este um espaço importante para o desenvolvimento das práticas promoção da saúde.

O estudo se concretizou em Florianópolis, cidade com 433.185 residentes, em unidades de saúde da rede de atenção primária à saúde; e em Toronto, cidade com mais de 2,5 milhões de habitantes, em CHC, com o intuito de compreender e comparar as práticas de promoção da saúde.

Em Florianópolis, o estudo ocorreu em cinco unidades de saúde. Dentre as unidades dos Distritos Sanitários, selecionaram-se as que tinham o maior número de ESF e atuação do NASF. Com a intenção de contemplar o maior número de profissões atuantes na atenção primária à saúde do município, foi sorteada uma equipe do NASF para integrar a pesquisa

Em Toronto, a pesquisa foi realizada em dois CHC, escolhidos após uma análise de suas principais características, uma vez que desenvolvem diversos projetos de promoção da saúde e são comparáveis em tamanho e estrutura aos centros analisados no estudo de Florianópolis.

A coleta de dados realizada em Toronto foi entre os meses de abril e junho de 2014 e, em Florianópolis, entre julho e outubro de 2015, baseando-se em entrevistas semiestruturadas com os profissionais das unidades de saúde, com questões guias, relacionadas ao tipo de formação recebida para realizar as atividades; às práticas que são desenvolvidas e sua frequência; às estratégias metodológicas utilizadas; às facilidades, dificuldades e resultados pertinentes à promoção da saúde; e à atuação sobre os determinantes sociais da saúde. As entrevistas tiveram duração aproximada de uma hora, sendo realizadas em Florianópolis pelos autores (C.G.R. e C.C.C.), e em Toronto, por (I. T.S.B.H., E.F. e D.G.), e pela pesquisadora Jennifer Ko.

Em Florianópolis, foram entrevistados 25 profissionais de nível superior das ESF e do NASF, sendo cinco médicos da saúde da família, um pediatra, cinco enfermeiros, cinco odontólogos, um nutricionista, um farmacêutico, um fisioterapeuta, um assistente social e cinco gestores. Excluindo-se os de nível técnico e agentes comunitários por não atenderem o critério de possuir graduação, mas não menos importantes no desenvolvimento das práticas de promoção da saúde. Todas as entrevistas foram realizadas nos consultórios das unidades de saúde, em ambiente privativo e silencioso, com data e horário marcados.

Em Toronto, foram entrevistados dez profissionais de nível superior atuantes nos dois CHC, sendo dois enfermeiros, dois médicos, dois nutricionistas, dois promotores de saúde e dois gestores. Como em Florianópolis, as entrevistas foram realizadas em sala privativa no próprio Centro. Foi convidado um número semelhante de participantes em ambas as cidades. Foram incluídos os profissionais que desenvolviam práticas de promoção da saúde, independentemente de categoria profissional, mas com graduação e indicados pela gerência dos CHC e unidades de saúde. As entrevistas foram gravadas digitalmente.

Para a análise dos dados, valeu-se da análise temática de Minayo 22, que se desdobra em três momentos: pré-análise, exploração do material, e tratamento dos resultados obtidos com sua interpretação. O referencial teórico que orientou a análise dos dados foi o da promoção da saúde e determinantes sociais da saúde 2,5,23. A pré-análise consistiu da transcrição fiel das entrevistas gravadas, leituras do material e organização dos dados. As transcrições foram armazenadas em duas pastas de arquivos do serviço de armazenagem virtual, organizadas pelo país de coleta. Esses documentos contemplam as falas dos sujeitos entrevistados, as notas dos entrevistadores, duração da entrevista, horário e local. 
Nesse movimento inicial, objetivou-se estabelecer a primeira classificação dos dados. Organizaram-se as informações com base nos objetivos que nortearam a pesquisa, procurando estabelecer uma primeira aproximação com os significados revelados nas falas dos sujeitos.

Após a leitura exaustiva do material transcrito das entrevistas, capturaram-se as ideias centrais do tema em questão. Os temas centrais foram formados baseando-se no sentido principal das perguntas feitas pelos pesquisadores, num total de três tipos: práticas localizadas, facilidades e dificuldades para realizar as práticas de promoção da saúde, buscando compreender as orientações teóricas que as direcionavam.

Com a definição dos temas, seguiu-se para a terceira etapa, tratamento dos resultados, na qual foi estabelecido um código de siglas e números que representavam as iniciais da unidade e o codinome escolhido pelo entrevistado em cada entrevista, sendo copiadas e coladas as falas principais em quatro planilhas no serviço de armazenagem virtual. Relacionadas as entrevistas com cada tema central, confrontaram-se as diferentes falas e construiu-se a análise final dos dados.

$O$ rigor da análise foi mantido da seguinte maneira: em Toronto, os dados foram analisados por duas pesquisadoras (Jennifer Ko, D.G.) e discutidos pelo grupo. Uma vez finalizado o esquema de codificação, o mesmo foi aplicado à totalidade dos dados. Esse processo de verificação permitiu a elaboração de um sistema de análise que foi utilizado para examinar os dados em Florianópolis, porém, ampliado para contemplar as realidades locais como: a atuação do NASF, os grupos de educação em saúde e a abrangência territorial. Modificações essas feitas pelas pesquisadoras (I.T.S.B.H. e C.C.C.).

A pesquisa foi aprovada pelo Comitê de Ética em Pesquisa da Universidade de Toronto sob o no 29961. No Brasil, a aprovação foi da Secretaria Municipal de Saúde de Florianópolis e do Comitê de Ética em Pesquisa, sob o Parecer no 1.053.016, em conformidade com a Resolução no 466/2012 do Conselho Nacional de Saúde, envolvendo seres humanos. Em ambos os municípios, para manter o sigilo dos participantes, os mesmos foram denominados por codinomes escolhidos por eles próprios, e os $\mathrm{CHC}$ e unidades de saúde foram identificados por números. Os entrevistados, esclarecidos sobre a divulgação do estudo, assinaram o Termo de Consentimento.

\section{Resultados}

\section{Caracterização demográfica dos participantes}

Participaram do estudo 35 profissionais de saúde, 10 de Toronto e 25 de Florianópolis (Tabela 1). Desses, sete enfermeiros, oito médicos, cinco odontólogos, dois promotores de saúde, sete coordenadores, um assistente social, um farmacêutico, um fisioterapeuta e três nutricionistas. A faixa etária dos participantes variou de 24 a 60 anos. O tempo de formado variou de 1 a 30 anos. Registrou-se o tempo de atuação na atenção primária à saúde de 6 meses até 30 anos, e o na unidade de saúde variou de 6 meses a 30 anos. Destaca-se que em Toronto o tempo de atuação dos profissionais na atenção primária à saúde e nas unidades de saúde, em média de 3 anos, é consideravelmente superior ao tempo de atuação dos profissionais brasileiros, que é em média de 3 anos.

Em relação à formação em promoção da saúde, $60 \%$ dos profissionais afirmaram ter recebido algum tipo de treinamento durante a graduação ou em curso de especialização lato sensu ou stricto sensu. Em Florianópolis, os profissionais ressaltaram a especialização em saúde da família, oferecida pelo governo, como uma ferramenta de formação para a atuação sobre as práticas de promotoras de saúde; já em Toronto, os profissionais destacaram que existe curso de graduação específico de promoção da saúde.

\section{Práticas de promoção da saúde}

As práticas de promoção da saúde citadas pelos profissionais de Toronto demonstram a predominância das atividades de educação em saúde, tal qual foram relatadas pelos profissionais de Florianópolis. Porém, nota-se que em Toronto a multidisciplinaridade e os grupos de educação em saúde que abrangiam grupo de caminhada, de orientação sobre a hepatite $\mathrm{C}$, de alimentação saudável, de lideranças para discutir as demandas da comunidade, de homens, de mulheres, trabalhadores do sexo e usuários 
Tabela 1

Caracterização demográfica dos participantes. Florianópolis, Santa Catarina, Brasil, e Toronto, Ontário, Canadá, 2015.

\begin{tabular}{|c|c|c|c|c|}
\hline & \multicolumn{2}{|c|}{ Florianópolis } & \multicolumn{2}{|c|}{ Toronto } \\
\hline & $\mathbf{n}$ & $\%$ & $\mathbf{n}$ & $\%$ \\
\hline \multicolumn{5}{|l|}{ Profissão } \\
\hline Enfermeiro & 5 & 20 & 2 & 20 \\
\hline Médico & 5 & 20 & 2 & 20 \\
\hline Odontólogo & 5 & 20 & 0 & 0 \\
\hline Promotor de saúde & 0 & 0 & 1 & 10 \\
\hline Coordenador & 1 & 4 & 1 & 10 \\
\hline Assistente social & 1 & 4 & 2 & 20 \\
\hline Farmacêutico & 1 & 4 & 0 & 0 \\
\hline Fisioterapeuta & 1 & 4 & 0 & 0 \\
\hline Nutricionista & 1 & 4 & 2 & 20 \\
\hline \multicolumn{5}{|l|}{ Idade (anos) } \\
\hline Até 35 & 17 & 68 & 5 & 50 \\
\hline $36-50$ & 7 & 28 & 3 & 30 \\
\hline$>50$ & 1 & 4 & 2 & 20 \\
\hline \multicolumn{5}{|c|}{ Tempo de formação (anos) } \\
\hline Até 5 & 6 & 24 & 1 & 10 \\
\hline $5-10$ & 8 & 32 & 2 & 20 \\
\hline $11-20$ & 8 & 32 & 3 & 30 \\
\hline$>20$ & 3 & 12 & 4 & 40 \\
\hline \multicolumn{5}{|c|}{ Tempo de atuação na atenção primária (anos) } \\
\hline Até 1 & 3 & 12 & 0 & 0 \\
\hline $1-5$ & 9 & 36 & 1 & 10 \\
\hline $6-10$ & 6 & 24 & 4 & 40 \\
\hline$>10$ & 7 & 28 & 5 & 50 \\
\hline \multicolumn{5}{|c|}{ Tempo de atuação na unidade (anos) } \\
\hline Até 1 & 7 & 28 & 0 & 0 \\
\hline $1-5$ & 12 & 48 & 3 & 30 \\
\hline $6-10$ & 3 & 12 & 4 & 40 \\
\hline$>10$ & 3 & 12 & 3 & 30 \\
\hline
\end{tabular}

Fonte: elaborado pelos autores.

de drogas, de pais de crianças de zero a seis anos, de imigrantes recém-chegados e de idosos, estão mais presentes no cotidiano de trabalho:

"Eu também tenho um grupo de idosos toda terça-feira, e a ideia é envolvê-los em mais atividades em sua comunidade. A maneira como eu desenvolvo este grupo é perguntando-lhes o que eles gostariam de aprender" (Luna).

Em Florianópolis, os profissionais destacaram como atividades os grupos de gestantes, tabagismo, hipertensos e diabéticos, alimentação saudável, idosos e puericultura. As orientações no consultório sobre alimentação saudável, higiene, autocuidado, hepatite $\mathrm{C}$ e a prevenção de infecções bacterianas e virais também foram apontadas como forma de promover a saúde da população.

"A gente pratica promoção da saúde desde que o paciente se senta no consultório, perguntamos se ele tem uma alimentação saudável, pratica atividade física, fuma, como é a sua rotina diária e tudo isso faz parte da promoção da saúde" (Amanda).

Evidenciam-se as ações multidisciplinares voltadas para a gestão do estresse, exercício físico e orientação nutricional para diabéticos, atividades de baixo impacto e de redução de danos aos usuários de drogas, que no Brasil são alicerçadas pelo NASF. 
Em Toronto, ultrapassando os limites da orientação voltada para as práticas educativas, ressaltou-se um programa de promoção da saúde aos usuários de droga, intitulado drop-in. Esse programa tem o objetivo de acolher e criar vínculo com esses usuários, oferecendo café da manhã, chuveiro, lavanderia e computador na unidade de saúde. Baseando-se nesse vínculo são realizadas as ações de educação em saúde, conforme a necessidade que cada usuário apresenta. Os profissionais de Toronto demonstraram esclarecimento quanto ao conceito de determinantes sociais da saúde ao citar práticas de promoção que visam à equidade em saúde. Essas práticas são realizadas com base na territorialização como ferramenta para levantar os determinantes sociais da saúde de cada comunidade, suporte às famílias carentes que são realocadas para regiões suburbanas e a instrumentalização dos imigrantes no idioma nativo, que é o inglês, para que eles consigam se integrar à realidade.

A atuação sobre os determinantes sociais da saúde em Florianópolis abrange as parcerias intersetoriais com serviços que influenciam diretamente a qualidade de vida da população. Entretanto, essas parcerias estão ainda muito focadas na escola, no conselho tutelar, lares de idosos e organizações não governamentais, que são setores diretamente ligados às atividades das unidades de saúde e procuram responder a demandas pontuais. A intersetorialidade é bem consolidada em Toronto, visto que os profissionais relataram que realizam diversas intermediações com outros setores. Essas parcerias ocorrem por meio da transição de idosos das suas casas para as de repouso, de parcerias com as escolas, da assistência social, das empresas, do setor de habitação e contabilidade, farmácias e hospitais.

O que difere a atuação sobre a promoção da saúde nesses países é que, apesar de ambos terem um sistema de saúde universal, os CHC do Canadá priorizam suas atividades para as comunidades vulneráveis e, no Brasil, a ênfase da atenção primária à saúde é atender toda a população da sua área de abrangência.

\section{Facilidades para realizar as práticas de promoção da saúde}

Algumas facilidades para realizar as práticas de promoção da saúde destacadas pelos profissionais canadenses são semelhantes às dos profissionais brasileiros, porém, nota-se que eles entendem os determinantes sociais da saúde como elementos facilitadores, no momento em que citam a equidade, a diversidade cultural, o sistema de justiça social e o apoio da comunidade.

Diferentemente de Florianópolis, em Toronto existe a atuação do profissional promotor da saúde em vários $\mathrm{CHC}$, que auxilia na articulação das práticas de promoção nos Centros, como ressalta Jane: "Os promotores são facilitadores, pois trabalham com famílias jovens, usuários de drogas, imigrantes, membros da comunidade de lésbicas, gays, bissexuais e transsexuais".

Em relação ao acesso ao serviço de saúde, a visita domiciliar foi citada no Brasil como uma facilidade. As equipes se deslocam às casas dos clientes com o intuito de atender aos usuários impossibilitados de ir até o serviço de saúde. Os profissionais de Toronto salientaram o fácil acesso ao CHC com a marcação de consulta por telefone, e afirmaram que as pessoas conseguem afluir aos cuidados sempre que necessitam, o que ainda é relatado como um desafio no Brasil.

Ambos ressaltaram a sensibilização das equipes para a importância da atuação sobre as práticas de promoção e o engajamento dos profissionais ao realizarem essas atividades. Além disso, em Florianópolis, destacou-se o apoio da coordenação em relação à realização dessas práticas, bem como o trabalho multidisciplinar com o NASF nas unidades: "Os profissionais do NASF apoiam bastante as práticas de promoção da saúde. Eles fazem grupos dentro da unidade e facilitam o acesso das pessoas a essas atividades" (Clara).

\section{Dificuldades para realizar as práticas de promoção da saúde}

Os entrevistados de Toronto relataram que a falta do profissional promotor de saúde é vista como uma das dificuldades para realizar as práticas de promoção. Esse profissional possibilita a articulação e operacionalização dessas práticas: "Não temos promotores da saúde, o que é um grande desafio" (Byron). No cenário brasileiro, esse profissional não consta no quadro de trabalhadores da área da saúde. Diante disso, não foi apontada essa ausência como uma das barreiras para a realização das atividades de promoção. 
O processo de trabalho dos profissionais, de ambos os países pesquisados, tem o seu foco baseado na produtividade do cuidado, com o desenvolvimento de ações predominantemente individuais e curativas. Há uma cobrança dos gestores para que o atendimento da população ocorra por meio das consultas individuais, com pouca realização das práticas promotoras articuladas aos determinantes sociais da saúde, ocorrendo um desequilíbrio destas atividades de saúde. Conforme relatado por um profissional de Florianópolis:

"Atualmente eu só insiro a promoção da saúde dentro dos atendimentos individuais, não tenho participado de grupos ou atividades intersetoriais pela questão da demanda e a cobrança de consultas" (Orquídea).

Destacaram os entrevistados de Toronto que o trabalho clínico é visto como uma das principais dificuldades para a operacionalização das práticas de promoção da saúde. Os cuidados primários em Ontário são geralmente da responsabilidade dos médicos 19, como evidenciado nos relatórios anuais de várias CHC. Esses profissionais são treinados em intervenções individuais com base na atenção biomédica, e possuem formação limitada em promoção da saúde.

Em Florianópolis percebe-se, como uma das dificuldades, que há pouca compreensão do conceito positivo de saúde, ainda relacionado à mudança de estilos de vida e ao modelo preventivista, compreensão esta traduzida como medidas de prevenção, cura e reabilitação em saúde. Conforme identificado pelos entrevistados, o processo de trabalho em ambas as cidades permanece baseado no modelo biológico. Há pouco envolvimento e articulação da equipe, falta de organização para a realização de grupos e outras atividades de promoção da saúde. Além disso, os entrevistados afirmaram que politicamente há uma priorização para a realização de programas de cuidados primários, o que impede o desenvolvimento das práticas de promoção da saúde e sua articulação com os determinantes sociais da saúde. Ressaltaram, igualmente, a necessidade de cooperação intersetorial para abordar os determinantes sociais da saúde e promover a saúde da comunidade.

Outro ponto destacado em ambos os países como um desafio foi a questão da escassez de recursos humanos e materiais e a falta de processos avaliativos.

Em Toronto foram apontados diversos desafios relacionados à diversidade cultural e climática. Na descrição de Gerber, essas dificuldades envolvem desde o planejamento de um programa até o desenvolvimento das atividades do grupo, que muitas vezes não podem ser contínuas: "Há uma diversidade das línguas, onde os profissionais não conseguem se concentrar em todos ao mesmo tempo" (Gerber).

Os resultados da pesquisa demonstraram que o desenvolvimento das práticas de promoção da saúde na atenção primária á saúde é benéfico para os municípios estudados, apesar das facilidades, dificuldades e das diferenças e semelhanças entre eles como destacado na Tabela 2. Há a necessidade ainda de avanços, em ambos os municípios, para implantar as práticas de promoção da saúde, haja vista a continuidade do predomínio do modelo biomédico, ainda que a regulação da atenção primária à saúde esteja em desenvolvimento.

\section{Discussão}

A promoção da saúde, tanto no Canadá quanto no Brasil, tem contribuído para a mudança do pensamento sanitário moderno. Ao enfatizarem o papel dos determinantes sócias da saúde, no processo saúde/doença, resgatam o conceito positivo de saúde, contrapondo-se ao modelo preventivista, apesar das divergências e controvérsias. No Brasil, por meio da PNPS 5, é reafirmado o trabalho multidisciplinar como caminho para a atuação sobre a promoção da saúde, articulando os diversos atores em um determinado território, fortalecendo a assistência em saúde que corrobora com o eixo da reorientação dos serviços de saúde da Carta de Ottawa 2, que regula a promoção da saúde no Canadá.

No Brasil, o compromisso da promoção da saúde é ético do próprio SUS com a integralidade e a gestão participativa, uma vez que trata de instituir a atenção e gestão das políticas públicas em saúde que operem na indissociabilidade entre a clínica e a promoção. Os recursos financeiros para o desenvolvimento das práticas de promoção foram implantados em diversos programas como, por exemplo, redução do tabaco e uso do álcool, alimentação saudável, que evidenciam a consolidação da PNPS, mas com predominância ainda da dimensão preventivista 3 .

Isso não difere do Canadá, onde a promoção da saúde ainda permanece fortemente estruturada no paradigma de atenção biomédico, com programas desenvolvidos com base no modelo 


\section{Tabela 2}

Análise comparativa acerca das práticas de promoção da saúde realizadas e as principais facilidades e dificuldades em realizar estas práticas na atenção primária. Florianópolis, Santa Catarina, Brasil, e Toronto, Ontário, Canadá, 2015.

\begin{tabular}{|c|c|c|}
\hline Práticas & Florianópolis & Toronto \\
\hline Práticas de promoção da saúde & $\begin{array}{l}\text { Grupos de educação em saúde; palestras; } \\
\text { ações multidisciplinares; orientação em } \\
\text { consultório; territorialização; parcerias com } \\
\text { escolas, lares de idosos e organizações não- } \\
\text { governamentais. }\end{array}$ & $\begin{array}{l}\text { Grupos de educação em saúde; palestras; } \\
\text { ações multidisciplinares; programa } \\
\text { drop-in; territorialização; realocação de famílias } \\
\text { carentes; instrumentalização dos imigrantes } \\
\text { no idioma; transição de idosos para casas de } \\
\text { repouso; parceria com escolas, assistência } \\
\text { social, empresas, setor de habitação, } \\
\text { contabilidade, farmácia e hospitais. }\end{array}$ \\
\hline Facilidades para realizar as práticas & $\begin{array}{l}\text { Visitas domiciliares; engajamento profissional; } \\
\text { trabalho multiprofissional; sensibilização } \\
\text { da coordenação das unidades de saúde da } \\
\text { família; núcleo de apoio à saúde da família; } \\
\text { residência multiprofissional em saúde da } \\
\text { família. }\end{array}$ & $\begin{array}{c}\text { Determinantes sociais da saúde; promotores } \\
\text { da saúde; visitas domiciliares; acesso ao } \\
\text { serviço de saúde; intersetorialidade. }\end{array}$ \\
\hline Dificuldades para realizar as práticas & $\begin{array}{l}\text { Foco na produtividade de ações individuais } \\
\text { e curativas; pouca compreensão do conceito } \\
\text { positivo de saúde; pouco envolvimento e } \\
\text { articulação da equipe; escassez de recursos } \\
\text { humanos e materiais; falta de processos } \\
\text { avaliativos; intersetorialidade. }\end{array}$ & $\begin{array}{l}\text { Poucos promotores de saúde; foco na } \\
\text { produtividade de ações individuais e curativas; } \\
\text { pouca ênfase no trabalho multidisciplinar; } \\
\text { cuidados primários focados no médico; } \\
\text { pouco envolvimento e articulação da equipe; } \\
\text { escassez de recursos humanos e materiais; } \\
\text { falta de processos avaliativos; atuação frente à } \\
\text { diversidade cultural e climática. }\end{array}$ \\
\hline
\end{tabular}

Fonte: elaborado pelos autores.

epidemiológico de fatores de risco ${ }^{1}$. Embora esse país com sistema de saúde universal e inclusivo invista no fortalecimento da atenção primária, com enfoque comunitário 24 . Essa cooperação com a comunidade foi identificada em Toronto, já que a atuação interdisciplinar se mostra mais estruturada e orienta as ações de saúde.

No Brasil, a preocupação é voltada para a organização e estruturação de um sistema universal de saúde que supere a carência da abrangência nacional, de modo a endereçar às iniquidades sociais. Isso é identificado em Florianópolis, quando o trabalho multidisciplinar constitui a base da atenção primária á saúde, mas os profissionais pouco se articulam entre si, e com a atenção secundária e terciária, e por isto se mostram incapazes de prestar atenção continuada, ampliada e resolutiva à população 24 .

Outro ponto são as diferenças existentes e relacionadas ao contexto socioeconômico em que se desenvolvem as práticas de promoção da saúde desses países e se reproduzem nas cidades estudadas. O Canadá, nação rica e desenvolvida, com sistema de proteção social e democracia sólida, preocupase em manter a qualidade do sistema de saúde, reduzindo custos, e obter êxito frente aos desafios da globalização. O Brasil, país com economia média, alta concentração de renda, sociedade autoritária e com muitas desigualdades sociais, tem no SUS uma possibilidade de fortalecimento da cidadania e efetivação de políticas sociais 25 .

A inserção dos determinantes sócias da saúde com a promoção da saúde constitui outro espaço vital para a melhoria da atenção em saúde. Em Toronto, identificou-se sinergia entre a promoção da saúde e os determinantes sociais da saúde quando buscam ampliar o acesso da população, especialmente os imigrantes, ofertando serviços essenciais, como saúde, educação e habitação. A sustentabilidade do trabalho com os determinantes sociais da saúde passa obrigatoriamente pela parceria sólida, intersetorialidade com outros setores da comunidade 26,27 que em Florianópolis carecem de fortalecimento. 
A integração da promoção da saúde e os determinantes sócias da saúde ainda é vista como um desafio, uma vez que o foco no estilo de vida individual continua a ser atraente para atores políticos dominantes. A cooperação entre a promoção da saúde e os determinantes sociais da saúde possibilitaria ações de mudança sobre a política e mudança socioambiental, de redistribuição, de ações em torno da sustentabilidade e do ecossistema, justiça social e de desenvolvimento da sociedade, estimularia a compreensão dos determinantes individuais, coletivos e sociais 10 .

Em ambas as realidades prevalece o desenvolvimento das práticas de promoção da saúde em grupos, e estas limitadas a identificar os efeitos prejudiciais de determinados comportamentos de vida. Geralmente atuam normatizando estilos de vida, mantendo o foco na doença e na eliminação de hábitos tidos como "de risco" 28 . É mister avançar com programas e iniciativas de promoção da saúde que busquem o fortalecimento dos sujeitos e comunidade, numa perspectiva crítica, e potencializar processos de transformação, assegurar a autonomia das pessoas sobre a realidade social 29.

Urge esforços para fortalecer as ações da promoção da saúde em todos os serviços de saúde e em todos os níveis. A efetividade desses princípios depende em nível macro da atuação sobre os determinantes sociais e, em nível micro, sobre a pessoa, família e comunidade. No entanto, prevalece como corrente hegemônica de promoção a abordagem tradicional voltada para mudanças de "estilos de vida saudável”, com a culpabilização dos usuários pelos profissionais 13 .

Uma questão essencial para a mudança desse enfoque preventivista seria o diálogo com outras políticas e setores da sociedade, incluindo o privado e a sociedade civil. Ratifica-se a necessidade de promover uma educação permanente e de capacitação em promoção da saúde e determinantes sócias da saúde, a fim de ampliar o engajamento e a reflexão crítica dos gestores e trabalhadores, e fortalecer o desenvolvimento sustentável 30 .

A promoção da saúde deve ser vista como um campo dinâmico e complexo, pois em ambas as realidades e em outros estudos 31 há predominância do paradigma flexneriano, sendo hegemônico no campo da saúde.

Em parte isso ocorre porque a saúde e o bem-estar são determinados por fatores que estão fora do sistema de saúde, e incluem desigualdades relacionadas aos aspectos políticos e socioeconômicos, novos padrões de consumo associados à alimentação, comunicação e mudanças demográficas. A promoção da saúde emerge com a possibilidade de inovar essas condições complexas dos níveis familiar e comunitário ao buscar a implantação do conceito ampliado de saúde 32.

A promoção da saúde busca superar o modelo biomédico ao adotar uma compreensão ampliada de saúde que requer ação intersetorial e engajamento comunitário, como forma de reduzir as vulnerabilidades e riscos 32. Para o seu enfrentamento, necessita utilizar os cinco campos de ação propostos na Carta de Ottawa 2 e as três estratégias: advocacy, mediação e capacitação.

Como limites, destaca-se que os resultados se referem a contextos específicos de dois municípios, portanto, os dados não podem ser generalizados para o Brasil e o Canadá. Além disso, existe a dificuldade de compreender as diferenças e semelhanças entre municípios com sistemas de saúde universal, mas que apresentam especificidades próprias que nem sempre eram visíveis e lógicas.

\section{Considerações finais}

Com base neste trabalho consegue-se visualizar as convergências e divergências entre as equipes de saúde da atenção primária à saúde em duas cidades de dois países com contextos socioeconômicos distintos acerca das práticas de promoção da saúde. Evidenciou-se que em ambas as realidades essas práticas estão muito direcionadas para as atividades de grupos e ações educativas, reforçando a culpabilização dos indivíduos que os instiga à mudança de estilos de vida. Esse enfoque acaba por não abranger os demais campos de ação da promoção da saúde e há pouca articulação com os determinantes sócias da saúde. A atenção à saúde ainda se mostra preventivista e com ênfase na promoção behavorista. Acredita-se que isso se deva à formação voltada ao modelo hegemônico biologicista dos profissionais.

No Brasil, já existem avanços referentes às políticas públicas voltadas especificamente para a promoção da saúde, como a PNPS. Diferentemente, o Canadá amplia a atuação dos profissionais sobre os determinantes sócias da saúde e a intersetorialidade, e tem na composição de suas equipes a figura do promotor da saúde que atua especificamente nas ações de promoção. 
Como possibilidade para a implementação das práticas de promoção da saúde são imprescindíveis as parcerias intersetoriais. Percebe-se a limitação dos profissionais de Florianópolis em realizar essas parcerias porque geralmente são atreladas a outros serviços que integram o setor saúde, como hospitais e secretarias. Já em Toronto se mostra mais ampla, abrangendo diversos setores da sociedade.

Ressalta-se a importância de ampliar a compreensão das práticas de promoção da saúde articuladas aos determinantes sócias da saúde, em especial dos gestores e formuladores de políticas públicas, visando à equidade, assim como impulsionar o desenvolvimento de novas pesquisas e proporcionar avanços na área da saúde.

\section{Colaboradores}

I. T. S. B. Heidemann, C. C. Cypriano, D. Gastaldo e S. Jackson foram responsáveis pela concepção do projeto, redação do artigo e aprovação da versão final a ser publicada. C. G. Rocha e E. Fagundes colaboraram na redação do artigo e aprovação da versão final a ser publicada.

\section{Agradecimentos}

À Coordenação de Aperfeiçoamento de Pessoal de Nível Superior. À Jennifer Ko, estudante de enfermagem da Lawrence S. Bloomberg Faculty of Nursing, University of Toronto, por sua participação na pesquisa.

\section{Referências}

1. Rootman I, Dupéré S, Pederson A, O’Neill M. Health promotion in Canada: critical perspectives on practice. 3 rd Ed. Toronto: Canadian Schoolars' Press Inc.; 2012.

2. World Health Organization. Ottawa charter for health promotion. Ottawa: World Health Organization/Health and Welfare Canada/ Canada Public Health Association; 1986.

3. Magalhães R. Avaliação da Política Nacional de Promoção da Saúde: perspectivas e desafios. Ciênc Saúde Coletiva 2016; 21:1767-76.

4. Malta DC, Moraes Neto OL, Silva MMA, Rocha D, Castro AM, Reis AAC, et al. Política Nacional de Promoção da Saúde (PNPS): capítulos de uma caminhada ainda em construção. Ciênc Saúde Coletiva 2016; 21:1683-94.

5. Secretaria de Vigilância em Saúde; Secretaria de Atenção à Saúde, Ministério da Saúde. Política Nacional de Promoção da Saúde: PNPS: revisão da Portaria MS/GM no 687, de 30 de março de 2006. Brasília: Ministério da Saúde; 2015.

6. Ferreira Neto JL, Kind L, Resende MCC, Colen NS. Processos da construção da Política Nacional de Promoção da Saúde. Cad Saúde Pública 2013; 29:1997-2007.

7. Heidemann ITSB, Wosny AM, Boehs AE. Promoção da saúde na atenção básica: investigação temática de Paulo Freire. Ciênc Saúde Coletiva 2014; 19:3553-9.

8. McManus A. Health promotion innovation in primary health care. Australas Med J 2013; 6:15-8.

9. Robertson G. 2017: a new era for health promotion or just another year? Glob Health Promot 2017; 24:3-4.

10. Jackson SF, Birn AE, Fawcett SB, Poland B, Schultz JA. Synergy for health equity: integrating health promotion and social determinants of health approaches in and beyond the Americas. Rev Panam Salud Pública 2013; 34:473-80.

11. Popay J, Whitehead M, Carr-Hill R, Dibben C, Dixon P, Halliday E, et al. The impact on health inequalities of approaches to community engagement in the new deal for communities regeneration initiative: a mixed-methods evaluation. Public Health Res 2015; 3:1-178. 
12. Pettersson B. Some bitter-sweet reflections on the Ottawa Charter commemoration cake: a personal discourse from an Ottawa rocker. Health Promot Int 2011; 26:173-9.

13. Norman AH, Tesser CD. Acesso ao cuidado na Estratégia Saúde da Família: equilíbrio entre demanda espontânea e prevenção/promoção da saúde. Saúde Soc 2015; 24:165-79.

14. Senado Federal. Constituição da República Federativa do Brasil de 1988. Brasília: Centro Gráfico do Senado Federal; 1988.

15. Durand MK, Heidemann ITSB. Promoção da autonomia da mulher na consulta de enfermagem em saúde da família. Rev Esc Enferm USP 2013; 47:288-95.

16. Ministério da Saúde. Política Nacional de Atenção Básica. Brasília: Ministério da Saúde; 2012. (Série E. Legislação em Saúde).

17. Sisson MC, Andrade SR, Giovanella L, Almeida PF, Fausto MCR, Souza CRP. Estratégia de Saúde da Família em Florianópolis: integração, coordenação e posição na rede assistencial. Saúde Soc 2011; 20:991-1004.

18. Home care in Canada 1999: an overview. http://www.hc-sc.gc.ca/hcs-sss/pubs/homedomicile/1999-home-domicile/index-eng. php\#a2 (acessado em 05/Jun/2017).

19. Marchildon GP, Hutchison B. Primary care in Ontario, Canada: new proposals after 15 years of reform. Health Policy 2016; 120:732-8.

20. Hutchison B, Glazier R. Ontario's primary care reforms have transformed the local care landscape, but a plan is needed for ongoing improvement. Health Aff (Millwood) 2013; 32:695-703.

21. Community health centres. https://www. cachc.ca/resources/reports/?did=1 (acessado em 17/Jun/2017).

22. Minayo C. Pesquisa social: teoria, método e criatividade. 29a Ed. Petrópolis: Editora Vozes; 2010.
23. Ministério da Saúde. Comissão Nacional sobre os Determinantes Sociais da Saúde. http:// www.determinantes.fiocruz.br/parceiros.asp (acessado em 20/Out/2016).

24. Faria LR, Alves CA. O cuidado na atenção primária à saúde: preliminares de um estudo comparativo Brasil/Canadá. Saúde Soc 2015; 24:72-85.

25. Carvalho SR. Saúde coletiva e promoção da saúde: sujeito e mudanças. 2a Ed. São Paulo: Editora Hucitec; 2007.

26. Pereira AS, Soares DKM, Oliveira KKA, Marques LA, Moreira DP, Vieira LJES. Análise da produção científica sobre os determinantes sociais da saúde. Cadernos ESP 2013; 7:40-52.

27. Dowbor TP, Westphal MF. Determinantes sociais da saúde e o Programa Saúde da Família no Município de São Paulo. Rev Saúde Pública 2013; 47:781-90.

28. Oliveira ES, Oliveira CR, Oliveira RC, Souza FS, Xavier IS. Política nacional de promoção da saúde e a prática de enfermagem: revisão integrativa. Rev Enferm UFPE on line 2014; 8:35-41.

29. Mendes R, Pezzato LM, Sacardo DP. Pesquisa -intervenção em promoção da saúde: desafios metodológicos de pesquisar "com". Ciênc Saúde Coletiva 2016; 21:1737-46.

30. Tavares MFL, Rocha RM, Bittar CML, Petersen $\mathrm{CB}$, Andrade M. A promoção da saúde no ensino profissional: desafios na saúde e a necessidade de alcançar outros setores. Ciênc Saúde Coletiva 2016; 21:1799-808.

31. Tavares MFL. Promoção da saúde: a construção social de um conceito em perspectiva comparada. Cad Saúde Pública 2011; 27:1865-6.

32. Kumar S, Preetha GS. Health promotion: an effective tool for global health. Indian J Community Med 2012; 37:5-12. 


\section{Abstract}

The study aimed to compare the experiences with the organization of universal public healthcare systems in relation to health promotion in primary care units in Florianópolis, Santa Catarina State, Brazil, and Toronto, Ontario, Canada. This was a descriptive exploratory study with a qualitative approach in primary care units. Data were collected with semi-structured interviews containing questions on health promotion practices, with 25 health professionals in Florianópolis and 10 in Toronto. The data were discussed using thematic analysis, identifying the practices, difficulties, and facilities in health promotion. In the two cities, $60 \%$ of health professionals and health administrators had not received any specific knowledge on health promotion during their training. As for health promotion skills, health professionals in Toronto identified them with autonomy and social determinants, while in Florianópolis they were related to health education and community participation. In both cities, health promotion practices are targeted to individual and collective activities. The motivation to act comes from interdisciplinarity and the demands raised by the population. Health promotion is a relevant form of care and stimulus for individual and community autono$m y$, in light of social determinants. Such practices aim at comprehensive health for the community, but there are limits in the teams that still conduct disease-centered activities. Resources are limited, requiring inter-sector actions to improve quality of life. Healthcare centers on the hegemonic model, and progress is needed to achieve a positive approach to health and social determinants.

Health Promotion; Social Determinants of Health; Primary Health Care

\section{Resumen}

Comprender las experiencias de organización de los sistemas públicos de salud universales, respecto a la promoción de la salud en unidades de atención primaria de Florianópolis, Santa Catarina, Brasil, y Toronto, Ontario, Canadá. Se trata de una investigación exploratoria descriptiva de enfoque cualitativo, realizada en unidades de la atención primaria. Para la recogida de datos se utilizaron entrevistas semiestructuradas con cuestiones sobre las prácticas de promoción, con 25 profesionales en Florianópolis y 10, en Toronto. Los datos se discutieron mediante un análisis temático, identificando las prácticas, dificultades y facilidades de la promoción de la salud. En esas ciudades, un $60 \%$ de los profesionales y gestores no conocían específicamente las posibilidades de promoción en su formación. En lo que atañe a las habilidades promotoras de salud, en Toronto se identificó que los sujetos reconocen la autonomía y los determinantes sociales, ya en Florianópolis las relacionan con la educación en salud y participación popular. En ambas ciudades, las prácticas de promoción se dirigen a actividades individuales y colectivas. La motivación para actuar proviene de la interdisciplinaridad y de las demandas provenientes de la población. Se destaca la relevancia de la promoción, como forma de cuidado y estímulo a la autonomía del individuo y de la comunidad, considerando los determinantes sociales. Estas prácticas alcanzan la salud integral de la comunidad, no obstante, se observan los límites de los equipos que todavía realizan actividades dirigidas a la enfermedad. Los recursos son escasos, por lo que son necesarias acciones intersectoriales para la mejora de la calidad de vida. La atención a la salud está enfocada hacia el modelo hegemónico, careciendo avances hacia la concepción positiva de la salud y los determinantes sociales.

Promoción de la Salud; Determinantes Sociales de la Salud; Atención Primaria de Salud

Recebido em 19/Dez/2016

Versão final reapresentada em 28/Ago/2017

Aprovado em 02/Out/2017 